\title{
Tangence
}

\section{La mémoire des autres. Historiens et plagiaires d'Ancien Régime}

\section{The Memory of Others: Historians and Plagiarists of the Ancien Régime}

\section{Frédéric Charbonneau}

Numéro 74, hiver 2004

Édition critique et intertextualité. Réécritures, reprises, dérivations

URI : https://id.erudit.org/iderudit/009206ar

DOI : https://doi.org/10.7202/009206ar

Aller au sommaire du numéro

Éditeur(s)

Tangence

ISSN

1189-4563 (imprimé)

1710-0305 (numérique)

Découvrir la revue

Citer cet article

Charbonneau, F. (2004). La mémoire des autres. Historiens et plagiaires d'Ancien Régime. Tangence, (74), 59-69. https://doi.org/10.7202/009206ar
Résumé de l'article

Le XVIII ${ }^{\mathrm{e}}$ siècle a vu se renforcer progressivement la relation de l'auteur à son oeuvre et s'accuser corollairement le signe de l'infamie qui marque les plagiaires. On est encore loin pourtant de la sensibilité chatouilleuse aux emprunts qui caractérise notre époque. Des habitudes d'écriture et d'édition à la relation d'autorité, l'étude des Mémoires d'Ancien Régime permet de nommer et de mettre en rapport un certain nombre de facteurs qui compliquent à l'occasion le travail de l'érudit, au point parfois de rendre vain l'effort d'identification d'un auteur unique : la réversibilité du document et du monument, qui faisait des Mémoires, même les plus achevés, des sources pour d'autres ouvrages ; l'anonymat de la rédaction, sa délégation à des secrétaires, sa collégialité dans le cas particulier de certaines communautés religieuses ; et la fabrication ultérieure de l'oeuvre, par les libraires ou par les héritiers, à partir de textes hétérogènes. Ce sont moins là des faits que des problèmes, qu’il faut poser sinon résoudre, des espaces culturels à baliser ou à quadriller. 


\title{
La mémoire des autres. Historiens et plagiaires d'Ancien Régime
}

\author{
Frédéric Charbonneau, Université McGill
}

\begin{abstract}
Le $X V I I{ }^{e}$ siècle a vu se renforcer progressivement la relation de l'auteur à son œuvre et s'accuser corollairement le signe de l'infamie qui marque les plagiaires. On est encore loin pourtant de la sensibilité chatouilleuse aux emprunts qui caractérise notre époque. Des habitudes d'écriture et d'édition à la relation d'autorité, l'étude des Mémoires d'Ancien Régime permet de nommer et de mettre en rapport un certain nombre de facteurs qui compliquent à l'occasion le travail de l'érudit, au point parfois de rendre vain l'effort d'identification d'un auteur unique: la réversibilité du document et du monument, qui faisait des Mémoires, même les plus achevés, des sources pour d'autres ouvrages; l'anonymat de la rédaction, sa délégation à des secrétaires, sa collégialité dans le cas particulier de certaines communautés religieuses; et la fabrication ultérieure de l'œuvre, par les libraires ou par les héritiers, à partir de textes hétérogènes. Ce sont moins là des faits que des problèmes, qu'il faut poser sinon résoudre, des espaces culturels à baliser ou à quadriller.
\end{abstract}

Je prends mon bien où je le trouve.

Molière

Les problèmes que j'aimerais poser ici relèvent moins de la seule expérience de l'édition que du cadre plus général des études littéraires d'Ancien Régime, dont l'édition elle-même est évidemment tributaire. On sait que le XVIII ${ }^{e}$ siècle a marqué, dans le rapport de filiation entre l'auteur et l'œuvre, une époque de transition: celui-ci se resserre, il se singularise, ce dont témoignent aussi bien tels changements sémantiques enregistrés par les lexicographes que les lances rompues en faveur de la propriété littéraire par Diderot et consorts. Ainsi le terme d'originalité apparaît en français vers 1700 pour désigner ce qui est «inventé, imaginé sans 
modèle ou souvenir antécédent ${ }^{1}$ ", alors que l'original, dix ans plus tôt chez Furetière, n'est encore que ce qui sert de modèle à imiter ou à copier. Et s'il est entendu que le plagiaire, le «larron de pensées \& de livres ${ }^{2}$ », fut assez mal considéré de Martial à Cyrano de Bergerac, il est également certain qu'on en usait jadis sur cette matière avec moins d'acribie. D'un emploi limité d'abord à celui qui s'attribue les ouvrages d'autrui, le mot n'en vint à désigner que peu à peu celui qui pille les idées ou les expressions ${ }^{3}$. Dans une note critique relative à cette évolution, Julie Hayes remarquait d'ailleurs que différents articles de l'Encyclopédie tendent, pour ainsi dire, à décriminaliser le plagiat en le rapprochant de l'imitation traditionnelle ou en soulignant que la culture est avant tout bien commun ${ }^{4}$. L'auteur de l'entrée "Plagiarisme, ou selon d'autres, PlaGIAT», probablement Jaucourt ou Diderot lui-même, emprunte au Dictionnaire de Bayle (s.v. Éphore) des citations de Scudéry et de La Mothe le Vayer qui proposent une casuistique du larcin littéraire, et admet que l'on puisse piller les Anciens, à la rigueur les étrangers, mais point ceux de sa nation. À l'article "Plagiaire», qui suit immédiatement, l'auteur note que les Encyclopédistes, à l'instar des lexicographes, sont «exemts des lois communes du mien \& du tien. [...] Si nous dérobons [ajoute-t-il], c'est seulement à l'imitation des abeilles qui ne butinent que pour le bien public, \& l'on ne peut pas dire exactement que nous pillons les auteurs, mais que nous en tirons des contributions pour

1. Littré, Dictionnaire de la langue française, Paris, Gallimard et Hachette, t. V, 1959, s.v. original; voir également la définition que donne l'Encyclopédie: «ORIGINALITÉ, s. f. (Gramm.) maniere d'exécuter une chose commune, d'une maniere singuliere \& distinguée: l'originalité est très-rare», Encyclopédie, ou Dictionnaire raisonné des arts, des sciences et des métiers, New York, Pergamon Press, 1969, t. XI, p. 648.

2. Furetière, Dictionnaire universel, Paris, SLN et Le Robert, 1978, t. III, s.v. plagiaire.

3. Le terme latin plagiarius, dérivé du grec $\pi \lambda \alpha \gamma$ เos (oblique, équivoque, fourbe), désignait littéralement «celui qui vole les esclaves d'autrui, [ou] qui achète ou qui vend comme esclave une pers[onne] libre» (Gaffiot, Dictionnaire latin-français, Paris, Hachette, 1934, s.v.). Voir Roland de Chaudenay, Dictionnaire des plagiaires, Paris, Perrin, 1990, p. 27. Voir également Martial, Épigrammes, I, 52 ; Cyrano de Bergerac, "Contre La Mothe, brigand de pensées", "Contre Chapelle, brigand de pensées", Voyage dans la lune, suivi de Lettres diverses, édition de Maurice Laugaa, Paris, Garnier-Flammarion, 1970, p. 143-144.

4. Julie C. Hayes, «Imitation, copying, plagiarism: intricacies of textual transmission", Actes du huitième congrès international des Lumières, Bristol, 21-27 juillet 1991, Oxford, Voltaire Foundation, 1992, vol. II, p. 1007-1011. 
l'avantage des lettres». Mais c'est Marmontel, à l'article "Plagiat» du Supplément de 1777, repris plus tard dans ses Élémens de littérature, qui se prononcera avec le moins de détours en faveur de certaines appropriations :

C'est une sorte de crime littéraire, pour lequel les pédans, les envieux, et les sots ne manquent pas de faire le procès aux écrivains célèbres. Plagiat est le nom qu'ils donnent à un larcin de pensées; et ils crient contre ce larcin comme si on les volait eux-mêmes, ou comme s'il était bien essentiel à l'ordre et au repos public que les propriétés de l'esprit fussent inviolables. $[\ldots]$

Quiconque met dans son vrai jour, soit par l'expression, soit par l'à-propos, une pensée qui n'est pas à lui, mais qui sans lui serait perdue, se la rend propre en lui donnant un nouvel être, car l'oubli ressemble au néant. [...]

Dans le droit public, la propriété d'un terrein [sic] a pour condition la culture: si le possesseur le laissait en friche, la société aurait droit d'exiger de lui qu'il le cédât ou qu'il le fît valoir. Il en est de même en littérature; celui qui s'est emparé d'une idée heureuse et féconde, et qui ne la fait pas valoir, la laisse comme un bien commun, au premier occupant qui saura mieux que lui en développer la richesse ${ }^{5}$.

Propriété commune, la propriété littéraire? En même temps que les Modernes rompent avec l'esthétique de l'imitation pour accorder plus de crédit au génie, à l'inspiration personnelle et à la nouveauté, l'idéal collectif des Lumières, le caractère souvent collégial de leurs entreprises et certain impératif pragmatique ont peut-être joué en sens contraire, en mélangeant, pour ainsi dire, les espèces et en unissant les forces de chacun contre l'ennemi. Quoi qu'il en soit, il apparaît qu'à l'aube de la Révolution, l'appartenance exclusive d'une œuvre à son auteur n'est pas chose entièrement acquise - bien moins encore aux époques antérieures.

5. Jean-François Marmontel, «Plagiat», Élémens de littérature, dans Euvres, t. IV, Paris, Belin, 1819, p. 859-860. Voir Denis Diderot, Mémoire sur la liberté de la presse, édition de Jacques Proust, Paris, Éd. sociales, 1964, p. 42: «Je le répète, l'auteur est maître de son ouvrage, ou personne dans la société n'est maître de son bien. Le libraire le possède comme il était possédé par l'auteur. Il a le droit incontestable d'en tirer tel parti qui lui conviendra par des éditions réitérées. Il serait aussi insensé de l'en empêcher que de condamner un agriculteur à laisser son terrain en friche, ou un propriétaire de maison à laisser ses appartements vides. » 
Maints problèmes d'attribution que nous rencontrons dans notre pratique non seulement viennent de là, mais devraient être résolus par là. La relation d'autorité, de paternité littéraire, n'est pas simple fait positif que le chercheur pourrait exhumer au terme d'une patiente enquête: elle est un élément de l'intrigue qui enserre et traverse l'œuvre, et la rattache au monde; on ne l'atteindra qu'en son lieu propre, dans le réseau qui lui donne sens et portée. Les clefs et les diagnostics intemporels manquent trop souvent leurs objets: c'est pourquoi l'auteur véritable d'un passage de Catherine Bernard ou de l'abbé Raynal, une fois trouvé, me laisse insatisfait. Il importe avant tout de reconstituer au mieux les circonstances qui entourent l'élaboration des œuvres pour comprendre et décrire - plutôt que pour démystifier — les liens parfois si compliqués que les auteurs entretiennent avec elles.

Ainsi, le domaine des Mémoires d'Ancien Régime, auquel je m'intéresse depuis une dizaine d'années, présente, me semble-t-il, des traits susceptibles de débrouiller un peu cette problématique. Je rappelle quelques paramètres: à l'origine, les Mémoires sont une forme aristocratique du récit d'histoire, pratiquée dans une perspective apologétique et polémique. Plusieurs de ces mémorialistes ne tenaient pas eux-mêmes la plume: ils dictaient plutôt à des secrétaires, ou leur laissaient composer des archives — surtout des correspondances. En outre, il arrivait fréquemment - neuf fois sur dix - que les Mémoires fussent publiés de manière posthume, après être passés entre les mains de descendants et de libraires aux intérêts variés.

Ces faits appellent un certain nombres de remarques. En premier lieu, dans la mesure où ils relèvent du genre historique, les Mémoires visent à mieux faire connaître la chose publique, la res publica, et constituent de ce fait des documents utiles à l'édification de ce grand monument impersonnel qu'est l'Histoire nationale. Quelques mémorialistes - Henri, marquis de Beauvau par exemple - annoncent ainsi qu'on trouvera dans leurs Mémoires "assez d'éclaircissement des choses les plus notables, pour aider ceux à qui il en prendra envie d'en grossir une Histoire plus étenduë, \& plus methodique ${ }^{6} »$. Cela tient au caractère particulier, ou partiel, et brut de la plupart de ces textes, qui ne se sont pas entièrement émancipés de leur première fonction de fichiers ou de

6. Henri de Beauvau, Mémoires du Marquis de B ${ }^{* * *}$ concernant ce qui s'est passé de plus memorable sous le règne de Charles IV duc de Lorraine \& de Bar, s.l.n.d. [Metz, c. 1686], préface non paginée. 
brouillons. Cependant, alors même qu'ils atteignent en certains cas à la dignité d'œuvre, marquée chez un Furetière par l'emploi d'une majuscule initiale, alors que leur complexité et leur ampleur semble défier toute élaboration supplémentaire, les Mémoires ne sont pas pour autant à l'abri des historiographes, qui peuvent à leur gré les piller pour en nourrir des textes de commande ou pallier leur impéritie. Témoin Duclos: après avoir publié, en 1746, "une Histoire de Louis XI fortement inspirée des notes de l'abbé Legrand et qui contribua à son élection à l'Académie française ${ }^{7}$ », il fut nommé historiographe de France, charge dans laquelle il succédait à Voltaire (1750). L'année suivante, il faisait paraître ses Considérations sur les mours de ce siècle, puis des Mémoires pour servir à l'histoire des mœurs du XVIII siècle; enfin, une dizaine d'années plus tard, on sait qu'il travaillait à une histoire de la Régence, mieux connue sous le titre un peu racoleur de Mémoires secrets sur les règnes de Louis XIV et de Louis XV, qui ne devait être publiée qu'en 1791, près de vingt ans après sa mort. Or cette histoire - on s'en aperçut beaucoup plus tard, grâce à l'édition de 1830 des Mémoires de Saint-Simon —, n'était guère qu'un démarquage. L'historiographe, en effet, avait eu accès au Dépôt des Affaires étrangères, où les papiers de Saint-Simon étaient entrés par ordre de Choiseul en 1760, et il en avait fait, comme il l'écrit à Rousseau, des "extraits ${ }^{8}$ » qu'il a ensuite retranscrits, ce qu'il indique au demeurant dans sa préface:

Aussitôt que le roi m'eut nommé historiographe, mon premier soin fut de rassembler les pièces qui m'étaient nécessaires. J’ai eu la liberté d'entrer dans les différents dépôts du ministère, et j'en ai fait usage longtemps avant d'écrire. J'ai lu une infinité de Mémoires, et les correspondances de nos ambassadeurs. J'ai comparé les pièces contradictoires, et souvent éclairci les unes par les autres. Les Mémoires du duc de Saint-Simon m’ont été utiles pour le matériel des faits dont il était instruit; mais sa manie ducale, son emportement contre les princes légitimés et quelques gens en place, sont à un tel excès, qu'ils avertissent suffisamment d'être en garde contre lui.

7. François Fossier, "À propos du titre d'historiographe sous l'Ancien Régime», Revue d'histoire moderne et contemporaine, Paris, vol. XXXII, juillet-septembre 1985, p. 393.

8. Lettre du 17 décembre 1762, citée par Yves Coirault, «Le plagiaire malgré lui: Duclos "abréviateur" de Saint-Simon", dans R. Marache et H. Le Moal (sous la dir. de), Missions et démarches de la critique. Mélanges offerts au Professeur J. A. Vier, Paris, Klincksieck, 1974, p. 612. 
Puis il ajoute cette phrase, qui ne laisse pas d'inquiéter: "J'indiquerai mes sources lorsque le temps et les circonstances le permettront ${ }^{9}$.»

La méthode n'était pas nouvelle et l'éditeur remarquait, dans l'Avertissement de 1791, que Duclos avait «usé du droit qu'a tout écrivain de refondre les mémoires particuliers dans l'histoire générale ${ }^{10}$ ", fussent de tels mémoires supérieurs en tous points à de simples archives. On serait moins mal à l'aise si Duclos avait moins daubé sur le conseiller de la Régence, car la refonte en ce cas-ci est un véritable "pompage", dont les notes d'Yves Coirault, pour l'édition de Saint-Simon dans la Pléiade, révèlent toute l'étendue.

Ma deuxième remarque portera sur ce que Blandine Kriegel a nommé la "défaite de l'érudition ${ }^{11}{ }$. Les historiens en général, et les mémorialistes en particulier, surtout s'ils étaient nobles, étaient peu susceptibles de citer avec exactitude: c'eût été pédanterie de leur part. Excepté les mauristes, les membres de l'Académie des Inscriptions et ceux du Cabinet des Chartes, l'érudition ne trouve, sous les règnes de Louis XIV et de Louis XV, plus guère de défenseurs: Fénelon la condamne aussi bien que Voltaire, qui prétendra n'avoir eu d'autres sources, pour Le Siècle de Louis XIV, "qu'environ deux cents volumes de mémoires imprimés que tout le monde connaît ${ }^{12} »$. Le mémorialiste plus que tout autre est censé tenir son savoir de l'expérience: l'intérêt de son récit vient de ce qu'il est témoin oculaire. Aussi, s'il lui arrive de préciser d'où il tire telle information, c'est davantage pour afficher ses alliances que pour leur rendre ce qui leur est dû. Pillé par Duclos, Saint-Simon a lui-même exploité les mémoires manuscrits de Dangeau et de Torcy; or passant ses emprunts innombrables au premier sous silence, car il le méprisait, il rend hommage au second, homme de «beaucoup d'esprit, de probité, de lumières ${ }^{13}$ », son ami par surcroît:

9. Charles Pinot-Duclos, "Préface» des Mémoires secrets sur le règne de Louis $X I V$, la Régence et le règne de Louis XV, édition de F. Barrière, Paris, Firmin Didot, 1846, p. 13.

10. Simone Scott, "Saint-Simon et ses premiers lecteurs", Stanford French Review, Saratoga (Californie, É.-U.), vol. V, 1981, p. 43.

11. Blandine Kriegel, "La défaite de l'érudition », L'histoire à l'âge classique, Paris, Presses universitaires de France, t. II, 1988.

12. Voltaire, "Lettre à M. l'abbé Dubos", 30 octobre 1738, dans Euvres historiques, édition de René Pomeau, Paris, Gallimard, coll. «Bibliothèque de La Pléiade», 1957, p. 605-606.

13. Saint-Simon, Mémoires, édition d'Yves Coirault, Paris, Gallimard, coll. «Bibliothèque de La Pléiade», t. IV, 1985, p. 246. 
Torcy m'a prêté ses extraits. C'est d'où j'ai puisé le détail du récit que j'ai donné, depuis la mort du Roi, de la suite et du détail des affaires étrangères. Je les ai abrégés et n'ai rapporté que le nécessaire; mais ce qui s'est passé en 1718 m'a paru si curieux et si important que j'ai cru devoir, non pas abréger ni extraire, mais m'astreindre à copier fidèlement tout, et n'en pas omettre un seul mot. [...] [J]e n'ai pas voulu toutefois laisser ignorer au lecteur, si jamais ces Mémoires en trouvent, ce qui n'est pas de moi, par le mépris que j'ai pour les plagiaires, et lui donner en même temps la confiance la plus entière dans ce que je rapporte des affaires étrangères, en lui expliquant d'où je l'ai pris $[\ldots]^{14}$.

Il faut dire, et ce sera mon troisième point, que ce caractère inédit des Mémoires et des autres textes historiques les laissait disponibles, si je puis m'exprimer ainsi, durant une période plus ou moins prolongée: Dangeau, Torcy, Saint-Simon et Duclos marquent la règle à cet égard. C'est qu'une bonne histoire est, disait-on, un fruit exquis mais vert, et qu'il faut bien laisser mûrir. En effet, il pouvait être dangereux de publier certaines choses, surtout sur des contemporains: l'obscurité du cabinet, purgatoire nécessaire des écrits véridiques, permettait de laisser couler quelques générations et de transformer la mémoire individuelle ou collective en un passé désormais apaisé. Or Marmontel, lui-même auteur de Mémoires sur la régence $d u$ duc d'Orléans publiés de façon posthume (1805), comparait, on l'a vu, l'œuvre qu'on ne fait pas valoir au terrain demeuré en friche et sur lequel a droit le premier occupant, maxime juridique dont plusieurs se sont prévalus, semble-t-il: les faits et les événements ne sont-ils pas à tout le monde? Cela se complique encore lorsque l'identité de l'auteur n'est pas bien décidée. L'anonymat certes, autant que la publication posthume, est une prudence qui peut mettre en péril la propriété d'une œuvre. Mais les cas les plus difficiles sont ceux de Mémoires aux auteurs pluriels, pour lesquels on ne sait pas au juste qui a tenu la plume ni même qui a pris la responsabilité de la rédaction. Ces cas sont plus nombreux et plus variés qu'on ne croit. Ainsi, il est assez fréquent qu'un domestique - au sens ancien de personne attachée à une maison aristocratique: officier, intendant, secrétaire, favori - écrive de son propre chef des Mémoires pour servir à la vie de tel ou tel grand personnage : c'est

14. Saint-Simon, Mémoires, ouvr. cité, t. VII, 1987, p. 76-77. Voir Émile Bourgeois, La collaboration de Saint-Simon et de Torcy, Paris et Nogent-leRotrou, Daupeley, 1905. 
le cas de $\mathrm{M}^{\text {me }}$ de Motteville pour Anne d'Autriche, de Bois d'Ennemetz pour Gaston d'Orléans, etc. ${ }^{15}$. Cependant, il pouvait arriver que ce travail soit plus ou moins commandé: par exemple, $\mathrm{M}^{\mathrm{me}}$ de La Fayette note, dans son Histoire de Madame Henriette d'Angleterre, que la duchesse n'avait pas dédaigné de marquer de sa main certain endroit de cette vie qui l'intéressait à plus d'un titre:

L'année 1665, le comte de Guiche fut exilé. Un jour qu'elle me faisait le récit de quelques circonstances extraordinaires de sa passion pour elle: "Ne trouvez-vous pas, me dit-elle, que, si tout ce qui m'est arrivé et les choses qui y ont relation était écrit, cela composerait une jolie histoire? Vous écrivez bien, ajoutat-elle: écrivez, je vous fournirai de bons Mémoires ». [...]

Elle me conta la suite des choses qu'elle avait commencé à me dire $[\ldots]$; je lui montrais le matin ce que j'avais fait sur ce qu'elle m'avait dit le soir; elle en était très-contente. [...] [E]lle prit tant de goût à ce que j'écrivais, que, pendant un voyage de deux jours que je fis à Paris, elle écrivit elle-même ce que j'ai marqué pour être de sa main, et que j'ai encore ${ }^{16}$.

Dans ce cas précis, la demande est courtoise: $\mathrm{M}^{\text {me }}$ de La Fayette, bien qu'elle ne fût pas du rang de la princesse, était ellemême une dame de qualité, qui prend explicitement la responsabilité de son œuvre. Mais d'autres Mémoires sont rédigés par de simples secrétaires auxquels on en a donné l'ordre: ceux de Louis XIV, par exemple, dont on possède tout de même les minutes et certaines pages autographes, ou ceux du duc de Sully ${ }^{17}$, adressés à lui-même par quatre secrétaires, cas unique de Mémoires écrits à la deuxième personne. Et que faire de ces Mémoires écrits par des

15. Combinée à l'anonymat du rédacteur, cette écriture pour autrui peut mener à l'impasse: dans le cas, par exemple, des Mémoires dits de Gaston d'Orléans, généralement attribués à Algay de Martignac, au nom duquel est le privilège. Mais Georges Dethan a montré jadis que celui-ci n'en était que l'éditeur et il est parvenu à établir que le rédacteur était un certain Jean de Lasseré, qui n'assume nulle part l'énonciation, sinon négativement par de prudents gommages qui ont d'ailleurs permis de le retracer. Voir «L'auteur des Mémoires dits de Gaston d'Orléans ", XVII siècle, Paris, n 45,1959, p. 292297. À qui attribuer l'autorité de ces Mémoires, rédigés après la mort de Gaston? Doit-on la laisser à Martignac qui publiquement l'assume? Doit-on la donner à Lasseré?

16. Madame de La Fayette, Histoire de Madame Henriette d'Angleterre, édition de Gilbert Sigaux, Paris, Mercure de France, 1965, p. 20-21. Pour un exemple d'annotation de Madame, voir p. 78, note 2.

17 Louis XIV, Mémoires, B.n.F. Mss fr. 6732-6734; Sully, Mémoires, B.n.F. Mss fr. 10305-10314. 
spirituels ou par des religieuses, qui ne faisaient rien que par ordre de leurs directeurs de conscience ou de leurs abbesses? de ceux qui émanent de Port-Royal, par exemple, auxquels on s'est beaucoup intéressé ces dernières années ${ }^{18}$ ? Là, «[1]'auteur ne se réduit pas à l'écrivain [écrit à ce propos Francis Mariner] mais serait une sorte de collectivité ${ }^{19} »$. Ainsi les Mémoires de Pontis ont été élaborés par ordre de M. de Sacy puis rédigés par Pierre-Thomas Du Fossé, à la première personne comme s'il était lui-même Louis de Pontis, dont le consentement à l'entreprise avait été arraché par son directeur. "L'auteur des Mémoires de Pontis n'est ni le personnage dont le nom apparaît dans le titre ni l'écrivain qui narre l'histoire. L'auteur est celui qui autorise l'acte d'écrire et occupe la position clé dans le réseau énonciatif qui reflète les liens de dépendance spirituelle de l'Église ${ }^{20}$.»

Le cas le plus problématique reste toutefois celui des Mémoires de Richelieu, véritablement insoluble tant il est compliqué. Les manuscrits cyclopéens, dont une partie fut publiée sous ce titre au XIX ${ }^{\mathrm{e}}$ siècle, ont été rédigés par Harlay de Sancy à partir de la "montagne d'archives ${ }^{21}$ » produite par les bureaux du cardinal ministre - pièces diplomatiques et de politique courante, mémoires pour le conseil ; correspondances; relations d'ambassadeurs, de généraux, de secrétaires d'État, de chargés de mission; journaux, cahiers, diaires, etc. Impossible de savoir si le secrétaire a travaillé du vivant de Richelieu : on a prouvé la chose et son contraire. Christian Jouhaud rappelle à cet égard que l'érudit Robert Lavollée a consacré une bonne partie de sa vie au problème de l'écriture autographe du Cardinal, qui eût permis de reconnaître son intervention directe dans la genèse du manuscrit. Après des siècles de débats - Voltaire déjà contestait l'authenticité de l'Histoire de la mère et du fils qu'il attribuait à Mézeray ${ }^{22}$-, force est de conclure à une sorte de non-lieu: «[à] chaque fois, on

18. Outre de nombreuses rééditions (Mémoires de Fontaine par P. MengottiThouvenin, de Lancelot par D. Donetzkoff, de Du Fossé par S.-A. Vinci, de Pontis par A. Villard), on consultera les Actes du colloque sur Port-Royal et les Mémoires, Chroniques de Port-Royal, nº 48, Paris, 1999; et de Francis Mariner, Histoires et autobiographies spirituelles. Les Mémoires de Fontaine, Lancelot et Du Fossé, Tübingen, Gunter Narr, 1998.

19. Francis Mariner, Histoires et autobiographies spirituelles, ouvr. cité, p. 51.

20. Francis Mariner, Histoires et autobiographies spirituelles, ouvr. cité, p. 53.

21. Christian Jouhaud, "Les Mémoires de Richelieu: une logique manufacturière", Mots, Paris, $\mathrm{n}^{\circ}$ 32, septembre 1992, p. 81.

22. Voltaire, Le Siècle de Louis XIV, dans Euvres historiques, ouvr. cité, p. 1199. 
débouche sur le même constat de la certitude de la présence du Cardinal - à un moment ou à un autre - et, en même temps, de l'impossibilité de fixer et de saisir cette présence ${ }^{23}$.»

Enfin, et ce sera notre dernier point, les pratiques d'édition cavalières de l'époque contribuent à brouiller les cartes: en l'absence de l'auteur, puisqu'il s'agit de publications posthumes, le libraire bien souvent se donne la licence d'altérer le texte des œuvres selon les besoins du marché, par la censure ou par l'interpolation de morceaux étrangers. En agissant en quelque manière comme un auteur second, le libraire ajoute aux incertitudes déjà rencontrées des "plagiats» de son cru. Dans la mesure où les Mémoires forment un genre déjà contestataire, leur association avec des pièces franchement satiriques ou scandaleuses pouvait accroître leur attrait auprès d'un certain public, ce que savaient Pierre Marteau et autres imprimeurs supposés, comme en témoigne le cas des Mémoires de La Rochefoucauld. Selon les Segraisiana, ce serait Louis-Henri de Lomenie de Brienne qui les aurait transcrits sur la copie de Robert Arnauld d'Andilly pour les remettre ensuite au libraire Barthelin, à Rouen. La Rochefoucauld eut le temps de faire saisir cette contrefaçon, dont il ne reste pas d'exemplaire, ce qui n'empêcha pas l'ouvrage de paraître peu après à l'adresse fictive de Pierre van Dyck, à Cologne, dans une édition fautive, incomplète (première version tronquée des livres II, III, IV et VI), où l'on avait fondu des morceaux de Vineuil (La guerre de Paris) et de Saint-Évremond (La retraitte de M. de Longueville); en outre, si l'on en croit l'auteur dans son démenti, on y avait interpolé des passages injurieux pour la famille de Condé ${ }^{24}$.

Des habitudes d'écriture et d'édition au caractère de l'œuvre et de la relation d'autorité, ce premier tour d'horizon aura permis du moins de nommer et de mettre en rapport un certain nombre de facteurs qui compliquent à l'occasion le travail de l'érudit: la réversibilité du document et du monument; l'anonymat de la rédaction, sa délégation ou sa collégialité; et la fabrication

23. Christian Jouhaud, "Les Mémoires de Richelieu: une logique manufacturière", art. cité, p. 92.

24. Voir l'apparat critique de Louis Martin-Chauffier à son édition des Mémoires de La Rochefoucauld, dans Euvres complètes, Paris, Gallimard, coll. "Bibliothèque de La Pléiade», 1950 ; voir également notre "Ambivalence d'un duc et pair ", dans Recherches des jeunes dix-septiémistes, Actes du Ve colloque $d u$ CIR-17, Université de Bordeaux III, 28-30 janvier 1999, édités par Charles Mazouer, Tübingen, Gunter Narr, 2000, p. 229-239. 
ultérieure de l'œuvre à partir de textes hétérogènes. Ce sont moins là des faits que des problèmes, qu'il faut poser sinon résoudre, des espaces culturels à baliser ou à quadriller. S'il fallait en tirer le tracé d'avenues ouvertes à l'édition, peut-être pourrait-on envisager un apparat critique qui dépasse la seule identification des sources et aille jusqu'à l'analyse des procédés de réécriture et d'inclusion, et qui prenne acte des variations de statut de l'auteur et du texte dans le procès de sa genèse. Car enfin, ce que révèle cette rapide enquête est une étrange dérive de la mémoire personnelle, une désappropriation dans l'écriture de cette faculté pourtant si singulière, si intérieure et si intime, ainsi renvoyée à autrui, sans vergogne mais peut-être pas sans inquiétude, en des échappées qui interrogent les limites mêmes de soi. 